\section{Available at \\ www.ElsevierMathematics.com \\ POWERED B S CIENCE d DiREct.}

Ann. I. H. Poincaré - PR 40 (2004) 125-131

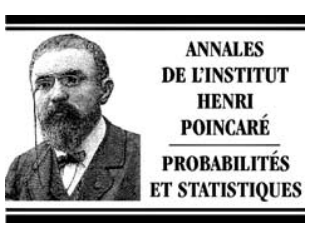

www.elsevier.com/locate/anihpb

\title{
On the covering by small random intervals
}

\author{
Ai-Hua Fan ${ }^{\mathrm{a}, \mathrm{b}, 1}$, Jun $\mathrm{Wu}^{\mathrm{a}, \mathrm{b}, 2}$ \\ ${ }^{a}$ Department of Mathematics, Wuhan University, Wuhan, Hubei, 430072, PR China \\ ${ }^{\mathrm{b}}$ LAMFA, CNRS, UMR 6140, University of Picardie Jules Verne, 33, rue Saint Leu, 80039, Amiens, France \\ Received 15 May 2002; accepted 15 July 2003
}

\begin{abstract}
Consider the random intervals $I_{n}=\omega_{n}+\left(0, \ell_{n}\right)$ (modulo 1 ) with their left points $\omega_{n}$ independently and uniformly distributed over the interval $[0,1)=\mathbb{R} / \mathbb{Z}$ and with their lengths decreasing to zero. We prove that the Hausdorff dimension of the set $\overline{\lim }_{n} I_{n}$ of points covered infinitely often is almost surely equal to $1 / \alpha$ when $\ell_{n}=a / n^{\alpha}$ for some $a>0$ and $\alpha>1$.

(c) 2003 Elsevier SAS. All rights reserved.
\end{abstract}

\section{Résumé}

Considérons des intervalles aléatoires $I_{n}=\omega_{n}+\left(0, \ell_{n}\right)$ (modulo 1$)$ dont les extrémités gauches $\omega_{n}$ sont indépendantes et uniformément réparties sur l'intervalle $[0,1)=\mathbb{R} / \mathbb{Z}$ et dont les longueurs décroissent vers zéro. Nous montrons que la dimension de Hausdorff de l'ensemble $\varlimsup_{\lim _{n}} I_{n}$ des points infiniment recouverts est presque sûrement égale à $1 / \alpha$ quand $\ell_{n}=a / n^{\alpha}$ avec $a>0$ et $\alpha>1$.

(c) 2003 Elsevier SAS. All rights reserved.

MSC: 60D05; 52C17; 28A80

Keywords: Random covering; Uniform sample spacing; Hausdorff dimension

\section{Introduction}

Let $\left\{\ell_{n}\right\}_{n} \geqslant 1$ be a sequence of positive real numbers which is decreasing to zero and let $I_{n}(\omega)=\left(\omega_{n}, \omega_{n}+\ell_{n}\right)$ (modulo 1) be a random interval where $\left\{\omega_{n}\right\}_{n} \geqslant 1$ is a sequence of independent random variables uniformly distributed over the unit interval $I=[0,1)$ which is identified with the circle $\mathbb{R} / \mathbb{Z}$. We consider the set $E_{\infty}(\omega)=$ $\overline{\lim }_{n} I_{n}$ of those points which are covered infinitely often.

It is easy to see that $E_{\infty}(\omega)$ is almost surely (a.s. for short) a set of Lebesgue measure 0 or 1 according to $\sum_{n=1}^{\infty} \ell_{n}<\infty$ or $\sum_{n=1}^{\infty} \ell_{n}=\infty$. A. Dvoretzky [2] asked the question when $E_{\infty}(\omega)=[0,1)$ a.s. or not. There was a series of contributions (for references and related topics see [5] for information before 1985 and [6]

\footnotetext{
E-mail addresses: ai-hua.fan@u-picardie.fr (A.-H. Fan), wujunyu@ @ublic.wh.hb.cn (J. Wu)

1 The author was partially supported by the Grant of Key Laboratory-Optimal Control and Discrete Mathematics.

2 The author is partly supported by the Special Funds for Major State Basic Research Projects of China.
} 
for recent information). A complete answer was obtained by L. Shepp [7]: $E_{\infty}(\omega)=[0,1)$ a.s. if and only if $\sum_{n=1}^{\infty}\left(1 / n^{2}\right) \exp \left(\ell_{1}+\ell_{2}+\cdots+\ell_{n}\right)=\infty$. It is the case for $\ell_{n}=a / n$ if and only if $a \geqslant 1$.

We address to the study of $E_{\infty}(\omega)$ when the covering intervals $I_{n}$ are small in the sense $\sum_{n=1}^{\infty} \ell_{n}<\infty$. As we mentioned above, in this case the set $E_{\infty}(\omega)$ is of Lebesgue measure zero. However, it is a.s. of second category in Baire sense (see [5, p. 55]). We will determine the Hausdorff dimension of $E_{\infty}(\omega)$ in the case $\ell_{n}=a / n^{\alpha}$ with $a>0, \alpha>1$.

Theorem. Suppose $\ell_{n}=a / n^{\alpha}$ for some $a>0$ and $\alpha>1$. Then

$$
\operatorname{dim} E_{\infty}(\omega)=\frac{1}{\alpha} \quad \text { a.s. }
$$

As we shall see from the proof of the theorem, $\ell_{n}=\mathrm{O}\left(n^{-\alpha}\right) \operatorname{implies} \operatorname{dim} E_{\infty}(\omega) \leqslant 1 / \alpha$ and $n^{-\alpha}=\mathrm{O}\left(\ell_{n}\right)$ implies $\operatorname{dim} E_{\infty}(\omega) \geqslant 1 / \alpha$. It follows that $\operatorname{dim} E_{\infty}(\omega)=1 / \alpha$ a.s. when the following limit exists:

$$
1<\alpha=\lim _{n \rightarrow \infty} \frac{-\log \ell_{n}}{\log n} .
$$

We point out that a similar result holds for random coverings on trees [3].

Back to the theorem. The inequality $\operatorname{dim} E_{\infty}(\omega) \leqslant 1 / \alpha$ is easy to see. It even holds for every $\omega$. Because $\left\{I_{n}(\omega)\right\}_{n \geqslant N}$ is a $\delta$-cover of $E_{\infty}(\omega)$ with $\delta=\ell_{N}$ and for any $\varepsilon>0$

$$
\sum_{n=N}^{\infty}\left|I_{n}\right|^{1 / \alpha+\varepsilon}=a^{1 / \alpha+\varepsilon} \sum_{n=N}^{\infty} n^{-1-\varepsilon \alpha}<\infty .
$$

In order to prove the inverse inequality, we will construct a random Cantor subset of $E_{\infty}(\omega)$ by using known results due to D.A. Darling on random spacings of uniform random samples. Before our proof of the theorem, let us give some preliminaries including Darling's results and a construction of Cantor set.

\section{Preliminaries}

Let $X_{1}, X_{2}, \ldots, X_{n}(n \geqslant 2)$ be a set of independent random variables uniformly distributed over the unit interval $I=[0,1)$. We call it a random sample of size $n$. Reordering the $n$ points $X_{1}, X_{2}, \ldots, X_{n}$ in their natural order from left to right, we get $n$ new random variables which will be denoted by $X_{(1)}, X_{(2)}, \ldots, X_{(n)}$. The intervals $\left[X_{(k)}, X_{(k+1)}\right), 0 \leqslant k \leqslant n$, are called the subspacings and their lengths are denoted by $L_{k}, 0 \leqslant k \leqslant n$ (by convention, $X_{(0)}=0$ and $\left.X_{(n+1)}=1\right)$. There is a vast literature on the distributions of $\left(L_{0}, L_{1}, \ldots, L_{n}\right)$ and related statistics. We will only need the following results among others due to D.A. Darling [1]. Suppose $h: I \rightarrow \mathbf{R}$. Let

$$
W_{n}=\sum_{j=0}^{n} h\left(L_{j}\right)
$$

The first two moments of $W_{n}$ are expressed by the following Darling formulas:

$$
\begin{aligned}
& \mathbb{E} W_{n}=n(n+1) \int_{0}^{1}(1-t)^{n-1} h(t) d t . \\
& \mathbb{E} W_{n}^{2}=n(n+1) \int_{0}^{1}(1-t)^{n-1} h^{2}(t) d t+n^{2}\left(n^{2}-1\right) \iint_{D}(1-x-y)^{n-2} h(x) h(y) d x d y,
\end{aligned}
$$


where $D=\{(x, y): x \geqslant 0, y \geqslant 0, x+y \leqslant 1\}$. We need to know how many subspacings with given length fall into a fixed subinterval. Let $J \subset I$ be a subinterval of length $\ell$ and let $0<s_{1}<s_{2}<1$. We denote by $M=M_{n}\left(\ell, s_{1}, s_{2}\right)$ the number of subspacings in $J$ whose lengths are between $s_{1}$ and $s_{2}$. Using the Darling formulas, J. Hawkes [4] obtained explicit expressions of the first two moments of $M$ :

$$
\begin{aligned}
& \mathbb{E} M=n \ell\left(\left(1-\frac{s_{1}}{\ell}\right)\left(1-s_{1}\right)^{n-1}-\left(1-\frac{s_{2}}{\ell}\right)\left(1-s_{2}\right)^{n-1}\right)+\left(1-s_{1}\right)^{n}-\left(1-s_{2}\right)^{n} . \\
& \mathbb{E} M^{2}=\mathbb{E} M+S\left(n, \ell, s_{1}\right)+S\left(n, \ell, s_{2}\right)-2 S\left(n, \ell,\left(s_{1}+s_{2}\right) / 2\right)
\end{aligned}
$$

where

$$
S(n, \ell, s)=n(n-1) \ell^{2}\left(1-\frac{2 s}{\ell}\right)^{2}(1-2 s)^{n-2}+2 n \ell\left(1-\frac{2 s}{\ell}\right)(1-2 s)^{n-1} .
$$

Proposition 1. Suppose $0<c_{1} \leqslant c_{2}<1 / 2,0<\ell<1$ and $n \geqslant 3$. Let $J \subset[0,1)$ be a subinterval of length $\ell$ and let $n$ be the sample size. Denote by $M$ the number of subspacings in $J$ having length in $\left[\frac{c_{1} \log n}{n}, \frac{c_{2} \log n}{n}\right]$. Then there exist constants $\gamma$ and $C$ only depending on $c_{1}$ and $c_{2}$ (independent of $\ell$ and $n$ ) such that

$$
\mathbf{P}\left(M<\gamma \ell n^{1-c_{1}}\right) \leqslant \frac{C}{n^{c_{2}-c_{1}}}
$$

for all $n$ such that $n^{1-2\left(c_{2}-c_{1}\right)} \ell \geqslant \log ^{4} n$.

Proof. We claim that

$$
\begin{aligned}
& \mathbb{E} M=\ell n^{1-c_{1}}+\mathrm{O}\left(\ell n^{1-c_{2}}\right) \\
& \mathbb{E} M^{2}=\mathbf{E} M+\ell^{2} n^{2\left(1-c_{1}\right)}+\mathrm{O}\left(\ell^{2} n^{2\left(1-c_{2}\right)}\right),
\end{aligned}
$$

where, and in the sequel, the constants involved in $\mathrm{O}(1)$ depend only on $c_{1}$ and $c_{2}$ and is independent with $\ell$ and $n$. First notice that

$$
\begin{aligned}
& \left(1-\frac{c \log n}{n}\right)^{n}=\frac{1}{n^{c}}\left(1+\mathrm{O}\left(\frac{\log ^{2} n}{n}\right)\right), \\
& \frac{1-\frac{c \log n}{\ell n}}{1-\frac{c \log n}{n}}=1+\mathrm{O}\left(\frac{\log n}{\ell n}\right), \\
& \left(\frac{1-\frac{c \log n}{\ell n}}{1-\frac{c \log n}{n}}\right)^{2}=1+\mathrm{O}\left(\frac{\log n}{\ell^{2} n}\right) .
\end{aligned}
$$

The equalities (8) and (9) hold under the condition $n \ell \geqslant c \log n$ which is ensured by the hypothesis made in the proposition. Let $s=\frac{c \log n}{n}$. Using (7) and (8), we get

$$
\left(1-\frac{s}{\ell}\right)(1-s)^{n-1}=\frac{1}{n^{c}}\left(1+\mathrm{O}\left(\frac{\log ^{2} n}{\ell n}\right)\right) .
$$

Then, by the formula (3), we obtain

$$
\mathbb{E} M=\ell n^{1-c_{1}}-\ell n^{1-c_{2}}+\mathrm{O}\left(n^{-c_{1}} \log ^{2} n\right)=\ell n^{1-c_{1}}+\mathrm{O}\left(\ell n^{1-c_{2}}\right) .
$$

Thus we have proved (5). Using (7) and (9), we get 


$$
\left(1-\frac{2 s}{\ell}\right)^{2}(1-2 s)^{n-2}=\frac{1}{n^{2 c}}\left(1+\mathrm{O}\left(\frac{\log ^{4} n}{\ell^{2} n}\right)\right) .
$$

Then

$$
S(n, \ell, s)=n^{2(1-c)} \ell^{2}+\mathrm{O}\left(\ell n^{1-2 c} \log ^{4} n\right) .
$$

Notice that $\ell n^{1-2 c} \log ^{4} n$ is dominated by $n^{2(1-c)} \ell^{2}$ if $n \ell \geqslant \log ^{4} n$. So the main term in $S(n, \ell, s)$ is $n^{2(1-c)} \ell^{2}$. Also notice that $\ell n^{1-2 c_{1}} \log ^{4} n$ is dominated by $n^{2\left(1-c_{2}\right)} \ell^{2}$ if $\ell n^{1-2\left(c_{2}-c_{1}\right)} \geqslant \log ^{4} n$ (this is the hypothesis). So we get (6).

As a consequence of (5) and (6), we have the following estimate of the variance of $M$ :

$$
\operatorname{Var}(M)=\mathbb{E} M+\mathrm{O}\left(\ell^{2} n^{2-\left(c_{1}+c_{2}\right)}\right)=\mathrm{O}\left(\ell^{2} n^{2-\left(c_{1}+c_{2}\right)}\right)
$$

By Chebyshev inequality,

$$
\mathbf{P}\left(M \leqslant \frac{\mathbb{E} M}{2}\right) \leqslant \mathbf{P}\left(|M-\mathbb{E} M|>\frac{\mathbb{E} M}{2}\right) \leqslant \frac{4 \operatorname{Var}(M)}{(\mathbb{E} M)^{2}}=\mathrm{O}\left(\frac{1}{n^{c_{2}-c_{1}}}\right) .
$$

Consider now a construction of generalized Cantor sets on $[0,1)$. Let $\left\{n_{k}\right\}_{k} \geqslant 1$ be a sequence of integers satisfying $n_{k} \geqslant 2$. Let $\left\{\rho_{k}\right\}_{k} \geqslant 1$ and $\left\{d_{k}\right\}_{k \geqslant 1}$ be two sequences of positive real numbers. Assume that for any $k \geqslant 1$, we have a collection $\mathcal{J}_{k}$ of closed subintervals of $[0,1)$. Each interval in $\mathcal{J}_{k}$ is called a $k$-interval. Suppose

(1) Each $k$-interval is of length $\rho_{k}$ and contains $n_{k+1}$ intervals;

(2) Each $(k+1)$-interval is contained in some $k$-interval;

(3) The gap between any two $k$-intervals is at least $d_{k}$.

Let $C_{n}=\bigcup_{J \in \mathcal{J}_{k}} J$ and $C_{\infty}=\bigcap_{k=1}^{\infty} C_{n}$. We call $C_{\infty}$ a generalized Cantor set.

Proposition 2. Consider the generalized Cantor set $C_{\infty}$ constructed above. Suppose that there is a number a $\geqslant 1$ such that $n_{k+1} d_{k+1} \geqslant \rho_{k}^{a}(\forall k \geqslant 1)$. Then we have

$$
\operatorname{dim} C_{\infty} \geqslant \liminf _{k \rightarrow \infty} \frac{\log \left(n_{1} n_{2} \cdots n_{k}\right)}{-a \log \rho_{k}}
$$

Proof. Define a probability measure $\mu$ on $[0,1)$ (concentrated on $C_{\infty}$ ) by

$$
\mu\left(J_{k}\right)=\frac{1}{n_{1} n_{2} \cdots n_{k}},
$$

where $J_{k}$ represents an arbitrary $k$-interval contained in $C_{k}$. Let $s$ be the liminf. Since $n_{1} n_{2} \cdots n_{k} \rho_{k} \leqslant 1$, we have $s \leqslant 1 / a \leqslant 1$. Suppose $s>0$. By the Frostman lemma, we have only to prove that for any $0<t<s$ and any open interval $U$ we have $\mu(U) \leqslant 2|U|^{t}(|U|$ denotes the length of $U$ ). Without loss of generality, we assume that $n_{1} n_{2} \cdots n_{k} \rho_{k}^{a t} \geqslant 1$ for all $k \geqslant 1$. Choose $k_{0}$ such that $\rho_{k_{0}+1} \leqslant|U|<\rho_{k_{0}}$. We distinguish two cases:

(a) The case $|U|<d_{k_{0}+1}$. Then $U$ intersects with at most one $\left(k_{0}+1\right)$-interval. So

$$
\mu(U) \leqslant \frac{1}{n_{1} n_{2} \cdots n_{k_{0}+1}} \leqslant \rho_{k_{0}+1}^{a t} \leqslant|U|^{a t} \leqslant|U|^{t} .
$$

(b) The case $|U| \geqslant d_{k_{0}+1}$. Then $U$ intersects with at most $\min \left(n_{k_{0}+1}, \frac{2|U|}{d_{k_{0}+1}}\right)\left(k_{0}+1\right)$-intervals. So 


$$
\begin{aligned}
\mu(U) & \leqslant \frac{1}{n_{1} n_{2} \cdots n_{k_{0}+1}} \min \left(n_{k_{0}+1}, \frac{2|U|}{d_{k_{0}+1}}\right) \\
& \leqslant \frac{1}{n_{1} n_{2} \cdots n_{k_{0}+1}} n_{k_{0}+1}^{1-t}\left(\frac{2|U|}{d_{k_{0}+1}}\right)^{t} \\
& \leqslant \frac{2}{n_{1} n_{2} \cdots n_{k_{0}}\left(n_{k_{0}+1} d_{k_{0}+1}\right)^{t}}|U|^{t} \leqslant \frac{2}{n_{1} n_{2} \cdots n_{k_{0}} \rho_{k_{0}}^{a t}}|U|^{t} \leqslant 2|U|^{t} .
\end{aligned}
$$

\section{Proof of theorem}

We only consider the case $\ell_{n}=a / n^{\alpha}$ with $a=1$. As we shall see, only the order $\alpha$ of $n^{\alpha}$ plays the role. So, we may also assume that $I_{n}$ is the closed interval $\omega_{n}+\left[0, \ell_{n}\right]$.

Fix two constants $0<c_{1}<c_{2}<1 / 2$ verifying the condition of Proposition 1 . Take a large integer $\Delta$. Define $m_{k}=\triangle^{k}(k=1,2, \ldots)$. For $k \geqslant 1$, let

$$
\begin{aligned}
\rho_{k} & =\ell_{2^{m_{k}+1}}, \quad d_{k}=\frac{c_{1} m_{k} \log 2}{2^{m_{k}}}, \\
n_{k} & =\left[\frac{\gamma \ell_{\rho_{k-1}} 2^{m_{k}\left(1-c_{1}\right)}}{2}\right]-1 \quad\left(\rho_{0}=1\right), \\
q_{k} & =1-C \frac{\prod_{j=1}^{k-1} n_{j}}{2^{m_{k}\left(c_{2}-c_{1}\right)}}
\end{aligned}
$$

where $[x]$ denotes the integral part of a real number $x$ and the constants $\gamma$ and $C$ are those in Proposition 1.

Consider the random sample of size $2^{m_{1}}$ from the uniform distribution over $[0,1): \omega_{2^{m_{1}}}, \omega_{2^{m_{1}}+1}, \ldots, \omega_{2^{m_{1}+1}-1}$. Applying Proposition 1 with $n=2^{m_{1}}$ and $\ell=1$. Proposition 1 is applicable if $\Delta$ is large enough so that $2^{m_{1}\left(1-2\left(c_{2}-c_{1}\right)\right)} \geqslant \log ^{4} 2^{m_{1}}$. We assume that $\gamma 2^{m_{1}\left(1-c_{1}\right)}>7$ so that $n_{1} \geqslant 2$. Let $\mathcal{L}_{1}$ be the set of left points of subspacing intervals contained in $J=[0,1)$ having length in $\left[\frac{c_{1} m_{1} \log 2}{2^{m} 1}, \frac{c_{2} m_{1} \log 2}{2^{m} 1}\right]$. By Proposition 1 , we have

$$
\mathbf{P}\left(\sharp \mathcal{L}_{1} \geqslant \gamma 2^{m_{1}\left(1-c_{1}\right)}\right)>1-\frac{C}{2^{m_{1}\left(c_{2}-c_{1}\right)}}=q_{1} .
$$

Thus with probability $q_{1}$ we can find a set $\mathcal{L}_{1}^{*} \subset \mathcal{L}_{1}$ with $n_{1}$ points such that for each point in $\mathcal{L}_{1}^{*}$ there is on its right side a point in $\mathcal{L}_{1} \backslash \mathcal{L}_{1}^{*}$. So, any two points in $\mathcal{L}_{1}^{*}$ has a distance at least $2 d_{1}$. Define

$$
C_{1}=\bigcup_{\omega \in \mathcal{L}_{1}^{*}}\left[\omega, \omega+\rho_{1}\right] .
$$

Notice that there are $n_{1}(\geqslant 2)$ intervals in $C_{1}$ each of which has length $\rho_{1}$ and that these intervals are separated by a distance at least $d_{1}$.

Suppose that with probability $q_{1} q_{2} \cdots q_{k}$ we have successively constructed a nested sequence of sets $C_{1} \supset C_{2} \supset$ $\cdots \supset C_{k}$ such that

(i) every $C_{j}(1 \leqslant j<k)$ is a union of disjoint closed intervals and each such interval in $C_{j}$ is of length $\rho_{j}$ and contains $n_{j+1}$ intervals contained in $C_{j+1}$, and every interval contained in $C_{j+1}$ is a subset of $C_{j}$;

(ii) the gap between two intervals contained in $C_{j+1}$ is at least $d_{j+1}$. 
We now construct $C_{k+1}$. Consider the random sample of size $2^{m_{k+1}}: \omega_{2^{m} m_{k+1}}, \omega_{2^{m} m_{k+1}+1}, \ldots, \omega_{2^{m_{k+1}+1}-1}$. This sample is independent of all preceding random samples in the construction of $C_{1}, C_{2}, \ldots, C_{k}$ since $2^{m_{k}+1}-1<$ $2^{m_{k+1}}$. Apply Proposition 1 to each interval $J$ contained in $C_{k}$ with $n=2^{m_{k+1}}$ and $\ell=\rho_{k}=\ell_{2^{m_{k}+1}}$. Notice that

$$
2^{m_{k+1}\left(1-2\left(c_{2}-c_{1}\right)\right)} \ell_{2^{m_{k}+1}}=2^{-\alpha+\Delta^{k}\left(\Delta\left(\left(1-2\left(c_{2}-c_{1}\right)\right)-\alpha\right)\right)} \geqslant \log ^{4} 2^{m_{k+1}}
$$

if $\Delta$ is large enough. So we can really apply Proposition 1. Thus if $\mathcal{L}_{k+1, J}$ denote the set of left points of subspacings contained in $J$ having length in $\left[\frac{c_{1} m_{k+1} \log 2}{2^{m_{k}+1}}, \frac{c_{2} m_{k+1} \log 2}{2^{m_{k+1}}}\right]$, we have

$$
\mathbf{P}\left(\sharp \mathcal{L}_{k+1, J} \leqslant \gamma \rho_{k} 2^{m_{k+1}\left(1-c_{1}\right)} \text { for some } J \subset C_{k}\right) \leqslant C \cdot \frac{\prod_{j=1}^{k} n_{j}}{2^{m_{k+1}\left(c_{2}-c_{1}\right)}} .
$$

In other words,

$$
\mathbf{P}\left(\sharp \mathcal{L}_{k+1, J}>\gamma \rho_{k} 2^{m_{k+1}\left(1-c_{1}\right)} \text { for all } J \subset C_{k}\right) \geqslant q_{k+1}
$$

where $J$ denotes a typical interval in $C_{k}$. For each $J$ in $C_{k}$, take a set $\mathcal{L}_{k+1, J}^{*}$ of $n_{k+1}$ points from $\mathcal{L}_{k+1, J}$ such that for each point in $\mathcal{L}_{k+1, J}^{*}$ there is on its right side a point in $\mathcal{L}_{k+1, J} \backslash \mathcal{L}_{k+1, J}^{*}$. Then construct

$$
C_{k+1}=\bigcup_{J \subset C_{k}} \bigcup_{\omega \in \mathcal{L}_{k+1, J}^{*}}\left[\omega, \omega+\rho_{k+1}\right]
$$

where $J \subset C_{k}$ means that $J$ is a component of $C_{k}$. Thus with probability $q_{1} q_{2} \cdots q_{k+1}$ we have constructed a nested sequence of sets $C_{1} \supset C_{2} \supset \cdots \supset C_{k+1}$ which have the properties described by (i) and (ii) (see above, $k$ being replaced by $k+1$ ). Thus by induction we get an infinite sequence of nested sets $C_{k}$ and we can construct a Cantor set $C_{\infty}=\bigcap_{k=1}^{\infty} C_{k}$ with probability

$$
p=\prod_{k=1}^{\infty} q_{k}>0 .
$$

The positivity of this probability is the consequence of

$$
\sum_{k=1}^{\infty}\left(1-q_{k}\right) \leqslant C \sum_{k=1}^{\infty} \frac{\prod_{j=1}^{k} n_{j}}{2^{m_{k+1}\left(c_{2}-c_{1}\right)}}<\infty
$$

because the general term of the series is bounded by

$$
\gamma^{k}\left(\prod_{i=1}^{k-1} \rho_{i}\right) 2^{\left(m_{1}+m_{2}+\cdots+m_{k}\right)\left(1-c_{1}\right)-m_{k+1}\left(c_{2}-c_{1}\right)}=\mathrm{O}\left(\gamma^{k} 2^{\Delta^{k}\left(\frac{1-c_{1}}{\Delta-1}-\left(c_{2}-c_{1}\right)\right)}\right) .
$$

By the construction, with probability $p>0$ we have $C_{\infty} \subset E_{\infty}(\omega)$. Actually $C_{\infty}$ is infinitely covered by those intervals $I_{n}$ with $2^{m_{k}} \leqslant n<2 \cdot 2^{m_{k}}$ for some $k \geqslant 1$.

Let us apply Proposition 2 to estimate the Hausdorff dimension of $C_{\infty}$ from below. Notice that $\rho_{k}=2^{-\alpha\left(\Delta^{k}+1\right)}$ and

$$
n_{k+1} d_{k+1} \approx m_{k+1} 2^{-\alpha m_{k}+\left(1-c_{1}\right) m_{k+1}-m_{k+1}}=\Delta^{k+1} 2^{-\Delta^{k}\left(\alpha+c_{1} \Delta\right)} .
$$

For any $a>1$ and small $c_{1}>0$ so that $c_{1} \Delta$ is small, the condition $n_{k+1} d^{k+1} \geqslant \rho_{k}^{a}$ is satisfied. Also notice that

$$
\lim _{k \rightarrow \infty} \frac{\log \left(n_{1} n_{2} \cdots n_{k}\right)}{-\log \rho_{k}}=\frac{1}{\alpha}\left(1-c_{1}\right) \frac{\Delta}{\Delta-1}-\frac{1}{\Delta-1} .
$$

Thus with probability $p>0$,

$$
\operatorname{dim} E_{\infty}(\omega) \geqslant \operatorname{dim} C_{\infty} \geqslant \frac{1}{a \alpha}\left(1-c_{1}\right) \frac{\Delta}{\Delta-1}-\frac{1}{\Delta-1} .
$$


Since $E_{\infty}(\omega)$ is a tail event, we have with probability one

$$
\operatorname{dim} E_{\infty}(\omega) \geqslant \frac{1}{a \alpha}\left(1-c_{1}\right) \frac{\Delta}{\Delta-1}-\frac{1}{\Delta-1} .
$$

Let $c_{1} \rightarrow 0, \Delta \rightarrow \infty$ and then $a \rightarrow 1$, we get $\operatorname{dim} E_{\infty}(\omega) \geqslant 1 / \alpha$ a.s.

\section{References}

[1] D.A. Darling, On a class of problems related to the random division of an interval, Ann. Math. Statistics 24 (1953) $239-253$.

[2] A. Dvoretzky, On covering a circle by randomly placed arcs, Proc. Nat. Acad. Sci. USA 42 (1956) 199-203.

[3] A.H. Fan, Oscillations of tree-indexed walks, LAMFA Prepublication, University of Picardie, 2000.

[4] J. Hawkes, On the asymptotic behaviour of sample spacings, Math. Proc. Cambridge Philos. Soc. 90 (2) (1981) $293-303$.

[5] J.P. Kahane, Some Random Series of Functions, second ed., in: Cambridge Stud. Adv. Math., vol. 5, Cambridge Univ. Press, Cambridge, 1985.

[6] J. Kahane, Random coverings and multiplicative processes, in: Fractal Geometry and Stochastics, II (Greifswald/Koserow, 1998), in: Progr. Probab., vol. 46, Birkhäuser, Basel, 2000, pp. 125-146.

[7] L. Shepp, Covering the circle with random arcs, Israel J. Math. 11 (1972) 328-345. 